\title{
Promoting psychological adaptation among navy sailors
}

\author{
Charles H Van Wijk $k^{97}$ \\ Institute for Maritime Medicine, Simon's Town \\ Jarred H Martin ${ }^{98}$ \\ Department of Psychology, University of Pretoria
}

\begin{abstract}
The mandate of the Institute for Maritime Medicine (IMM) is to support and enhance the operational performance of sailors of the South African Navy during maritime operations, while also ensuring positive long-term mental health outcomes of sailors who serve their country at sea. To achieve this, the IMM proposes to re-orientate the mobilisation and demobilisation programmes used for ship-based maritime operations towards a predict-and-promote (P\&P) approach, to enhance the psychological adaptation of sailors to the emotional demands of deployment as well as to support more adaptive forms of mental health resilience, both before and after sea-going operations.
\end{abstract}

First, this article aims to present the proposed P\&P approach for enhancing psychological adaptation during and after seaward deployments, with a specific focus on assessing personal emotional regulation (ER). For effective implementation, this approach is contingent on several clinical assumptions about ER in the operational environment, namely: the absence of significant psychopathology; the stability of the ER measure; the role of dispositional factors in operational adaptation; and the availability of population-specific normative data, which act as an interpretative guide of ER profiles for sailors. The second aim is to consider support for these assumptions, using previous experience during the mobilisation and/or demobilisation of ships involved in maritime operations. Support was found for all four assumptions, indicating the clinical and operational utility of the P\&P approach at the IMM broadly, and the assessment of ER for sailors in particular.

Keywords: adaptation, mental health promotion, navy deployments, resilience.

\section{Background}

The past decade has seen an increased operational tempo for the South African Navy (SAN), particularly in support of anti-piracy operations and maritime border security, in addition to regular training, international exercises, and diplomatic missions. It is well documented in deployment psychology studies that such operational demands typically require increased mental health support to military personnel in order to help them adjust to the personal and emotional demands of operational deployments, as well as integrating back into regular life at home after returning from operations. ${ }^{99}$ 
According to the doctrine of the South African Military Health Service, the Institute for Maritime Medicine (IMM) is responsible for providing maritime health support to the South African National Defence Force. ${ }^{100}$ By virtue of its geographical location in Simon's Town, the IMM is the primary point of maritime health support and service provision for the South African Navy Fleet. Included in the mandate of the IMM are the objectives to: support and enhance operational performance during maritime tasks (e.g. of sea-going units); and support adaptive long-term mental health outcomes of sailors who serve their country at sea.

One mechanism to achieve these objectives with sailors participating in maritime operations is to use the operational phases of mobilisation and demobilisation. The purpose of the involvement of military psychologists in mobilisation and demobilisation programmes is:

- $\quad$ to enhance psychological adaptation (PA) during missions (operationalised as the ability to fulfil a mission role, i.e. 'personal performance'); and

- $\quad$ to enhance PA after missions (operationalised as adaptive long-term mental health outcomes).

In short, IMM uses the institutional and operational mechanism of mobilisation and demobilisation both before and after operations to promote PA and enhance mental health resilience.

This promotion of PA for maritime operations takes the form of, accurate screening of SAN personnel, with the aim of identifying individuals potentially at risk of poor PA during operations; and timeous and appropriate streaming for targeted intervention, with the aim of mitigating the identified risk to effective PA. ${ }^{101}$

In this context, PAcan broadly be defined as an individual's ability to adjust to changes in their environment, in order to optimise personal functioning. This is particularly relevant in the psychology of isolated, confined and extreme (ICE) environments. ${ }^{102}$ ICE environments refer to settings characterised by hostile external conditions, exposure to a range of context-specific physical, mental and social stressors, and often require engineering technology to maintain human survival. ${ }^{4}$ ICE environments are, for instance, underwater habitats, spacecraft, remote weather stations, polar outposts, and of particular relevance here, ships at sea. Within ICE environments, PA is reflected by three indicators (the so-called 'Antarctic triarchy'), 4,103,104, namely:

- $\quad$ task ability (referring to the quality of work output);

- $\quad$ sociability (referring to the quality of interpersonal interaction); and,

- $\quad$ emotional stability (referring to the quality of internal self-regulation).

While all three indicators can be measured, it is a complex process to do so in ICE environments, and many have followed the route of choosing to measure a single factor that underpins all three indicators, for example emotional regulation. ${ }^{105}$ 
Emotional regulation (ER) refers to a "set of automatic and controlled processes involved in the initiation, maintenance and modification (i.e. 'regulation') of the occurrence, intensity and duration of feeling states".7,106,107,108 ER underpins personal performance across many aspects of daily life, such as family, work and sport. ${ }^{9}$ As such, it can be used to operationalise PA, in that individuals with more adaptive ER should be expected to manage their personal performance across work output, social interactions, and affective states effectively, especially under the psychological demands unique to ICE environments. ${ }^{7}$ In contrast, individuals with less adaptive ER are assumed to present with greater difficulties in managing their personal performance across these three indicators.

The aim of the rest of this article is two-fold. Firstly, it presents the approach followed by the IMM to the promotion of PA, in ICE contexts and after return to regular life, using the mobilisation and demobilisation programme for ship-based maritime operations. This approach is contingent on a number of assumptions, and effective implementation is subject to these assumptions being met. A second aim is to consider support for the assumptions, using previous experience during the mobilisation and/or demobilisation of ships involved in maritime operations.

\section{Predict and promote: A new approach towards supporting the psychological adaptation of SAN sailors}

Historically, various approaches to facilitate operational adjustment have been employed, including at the IMM. Preparation of sailors for their deployment (in the case of mobilisation) or return to home life (in the case of demobilisation) are typically done through one or more group-based presentations, where the deploying ship's company would be required to gather en masse to participate in the mobilisation and/or demobilisation programme. The programme then takes the form of sharing information on what to expect (before or after deployment), based on the assumption that advance knowledge will prepare individuals to cope with stressful situations should they occur. However, there are a number of limitations to this approach:

- $\quad$ such approach is based on the understanding that coping is situational, rather than (at least partially) dispositional;

- $\quad$ in the case of ship-based deployments, sailors are usually already serving on the vessel, have at least done multiple work-up trials at sea, and are thus familiar with both their specific tasks and the general routine on-board the ship; and

- $\quad$ there is little substantial empirical evidence available that this approach has much practical benefit to sailors or their families. ${ }^{109}$

Against the dearth of available evidence that this form of knowledge sharing through group presentations has beneficial effects on subsequent PA, the IMM proposed the adoption of a different strategy.

To achieve the promotion of operational adaptation - whether during or subsequent to a maritime mission - the IMM has developed a predict-and-promote (P\&P) approach, to be implemented as part of the mobilisation and demobilisation programme of shipbased maritime operations. 
Adaptation in ICE environments is indicated by the triarchy of (sustained) quality of work output, interpersonal interaction, and internal emotional stability, and ER facilitates adaptation in ICE environments in that it underpins this triarchy. As such, ER can be viewed as an indicator of psychological adaptation in context (conceptualised here as the ability to regulate internal responses to changes in that environment).

Therefore, within this framework, measurement of ER would enable the identification of risk for poor PA, both during mobilisation (for subsequent mission performance) as well as during demobilisation (for subsequent adverse mental health outcomes after return to regular life).

This would be accomplished through developing ER profiles using psychometric instruments. Within this framework, more or less adaptive forms of ER could then be visualised in the relationship between an individual's psychometric scale profile, and normative profiles for that naval population. For example:

- $\quad$ adaptive ER would be evidenced by psychometric scale profiles that lie within normal limits for that naval population, and which would indicate the ability to maintain PA better; and

- $\quad$ less adaptive (i.e. poorer) ER would be evidenced by psychometric scale profiles that deviate from normal (i.e. expected) profiles found in normative naval populations.

Therefore, screening of ER would support a P\&P approach, in that identification of high risk for less adaptive modes of ER would initiate automatic referral for intervention to enhance an individual's adaptation. ${ }^{110}$ Interventions would typically be short term and focused on enhancing PA, by, among others:

- $\quad$ identification and active monitoring of 'at-risk' personnel;

- $\quad$ enhanced personal preparation for deployment;

- fortifying more expeditious mechanisms of adaptive 'coping'; and/or

- development of situation-specific or circumstantial coping strategies, especially with regard to situations or circumstances that trigger less adaptive ER.

\section{Measurement of ER}

One commonly used psychometric tool that could support the measurement aspect of ER is the Brunel Mood State Scale (BRUMS), which is available in various configurations. In its original form, the BRUMS is a 24-item Likert-type self-report scale that measures transient affective mood states. ${ }^{11,112,113}$ It is used extensively internationally, and a substantial body of literature exists on its use in many domains (from sport performance to sleeping patterns to academic achievement). ${ }^{114,115,116}$ Published South African norms are available, making it convenient for local use. ${ }^{117}$ The scale is also widely used in clinical contexts, e.g. for intake baseline measurement and as a measure of the effectiveness of psychotherapeutic interventions. ${ }^{13}$ Pertinent to military deployments, the BRUMS has previously been able to predict self-report post- 
traumatic stress symptoms after maritime interdiction operations as well as to predict performance in ICE environments. ${ }^{118,119}$ Good concurrent and criterion validity has been reported internationally ${ }^{14,15}$ and locally. ${ }^{120}$

The BRUMS offers an effective screening of ER in two ways: firstly, by providing a current profile of mood states; and secondly, through its sensitivity to ER processes and mood state changes. As a result, less adaptive ER is more readily expected when profiles deviate from population norms and/or when the changes in ER profiles over time are characterised by a less normative scores profile. Deviations from expected profiles can be interpreted as suggesting less adaptive forms of self-regulation. This places individuals at the potential risk of poorer PA.

\section{Underlying assumptions}

The effective implementation of the recommended P\&P approach - that adaptation is facilitated by ER, and can reliably be predicted in ICE contexts - is contingent on a number of assumptions. The P\&P approach can thus only be meaningfully employed where these assumptions can be met, such as:

- the assumption of the absence of clinically significant and ER-compromising psychopathology;

- the assumption of the stability of the ER measure, e.g. that ER profiles at mobilisation (i.e. prior to deployment) are reliably predictive of ER profiles both during the deployment and at demobilisation (i.e. in the post-deployment period);

- the assumption that adaptation in ICE environments is (also) dependent on dispositional factors (e.g. dispositional resilience), and not purely dependent on specific circumstances such as mission-unique conditions; and

- the assumption of the availability of population-specific norms to serve as normative reference framework for the interpretation of ER profiles.

\section{Testing support for assumptions}

In order to consider implementation of the recommended P\&P approach, the rest of this article turns to evaluating support for the above-mentioned assumptions. It does so by examining previous experience during mobilisation and/or demobilisation of ships involved in maritime operations, using data from the IMM dataset of psychological measurement of sailors deploying to sea.

\section{Procedure}

IMM maintains a database for deploying sailors, consisting of general mental health screening data as well as mobilisation, mid-mission, and demobilisation ER data. The data come from actual missions, and were not collected as part of any prospective research study. This article draws from data generated during mobilisation and/or demobilisations programmes between 2015 and 2019. 


\section{Participants}

To consider the assumptions of the proposed approach, a total sample of 1057 participants ( $28,6 \%$ women, $71,4 \%$ men) were available, who provided data at any time point. The mean age of the total group was $30,6( \pm 6,6)$. Not all sailors provided data at all time points, and thus individual analyses may reflect different sample sizes.

\section{Measures}

A modified version of the BRUMS was administered at three time points - during mobilisation, mid-mission (typically $6-8$ weeks into a $12-14$-week mission), and again during demobilisation. The current 20 -item modified version used five mood states (i.e. excluding 'confusion') to calculate a Total Mood Distress score (TMD), which formed the profile for interpretation. The TMD ranges from -16 to +64 , with lower scores indicative of better ER. The BRUMS has been used extensively over the past eight years to screen for ER in deployment contexts, and considerable expertise exists at the IMM to interpret profiles in the context of maritime operations. Additionally, an existent dataset of BRUMS responses for the general Fleet $(\mathrm{N}=2382)$ was also available for comparison.

The Brief Sailor Resiliency Scale (BSRS) was administered during mobilisation. This scale measures dispositional resiliency across four domains of readiness, namely mental, physical, social and spiritual, and a comprehensive sailor resiliency score can be calculated, which was used in this analysis. ${ }^{3}$ Dispositional resilience refers to the personal quality that allows people to overcome hardships and even thrive in the face of it. ${ }^{121,122}$ It is usually considered an internal trait, developed throughout life, which allows an individual to work constructively through life's adversities, and is further considered a predictor of adaptation to stress/trauma, as well as to mental health. ${ }^{123,124}$

Clinical mental health screening data (collected during the biennial concurrent health assessment of SAN sailors) were available for 975 persons. This included markers of clinical psychopathology that would interfere with adaptive emotional regulation and impair performance across personal, social, and occupational spheres.

\section{Analyses}

The data were examined to:

- investigate the absence or presence of clinical psychopathology, by examining available mental health screening data that could be linked to the sample;

- investigate the stability of the BRUMS across three time points (by calculating correlational statistics), and the stability of the profile changes across time (by calculating the mean difference and its standard deviations between time points);

- investigate the role of mission circumstances (by using ANOVA), as well as a dispositional factor (by calculating correlational statistics) on ER profiles. 
Mission factors refers to the type of naval platform, type of mission, and mission duration, while the dispositional factor refers to resiliency; and

- $\quad$ provide normative reference data as baseline for future interpretation and comparison for the SAN Fleet (by developing population-specific means and standard deviations).

\section{Results}

All sailors of the SAN undergo a biennial general mental health screening (see Assumption 1, absence of clinical psychopathology). The available screening results for the period under study, i.e. 2015-2019 ( $\mathrm{N}=975)$ were reviewed by a clinical psychologist, who reported that the sample was free from ER-compromising clinical psychopathology. Further examination indicated that no single mental health marker was significantly correlated to any deployment measure at any time.

Regarding Assumption 2, stability of the ER measure - the stability of ER profiles across different times are presented in Tables 1 and 2. ER profiles of the participating sailors at mobilisation predicted ER profiles mid-mission and at demobilisation. Further, the results suggest that the profiles remained stable across the time periods. The variance of scores across time frames also appeared to remain stable, as did the variance in increases between time points. The narrow band of variance - at each time point, and in the increases between time points - further suggests that deviations may be easy to identify.

\begin{tabular}{|l|l|l|l|}
\hline Time point & N & $\mathbf{M}^{*}$ & SD \\
\hline Mobilisation TMD** & 458 & -7.8 & 6.4 \\
\hline Demobilisation TMD & 488 & -4.5 & 7.0 \\
\hline Mid-mission TMD & 111 & -5.8 & 7.5 \\
\hline Total fleet dataset TMD & $\mathbf{2 3 8 2}$ & $\mathbf{- 5 . 5}$ & $\mathbf{7 . 6}$ \\
\hline
\end{tabular}

Table 1. Normative ER profile data for SAN Fleet per time point.

$*=$ Mean

$* *=$ Total Mood Distress

\begin{tabular}{|l|l|l|l|l|l|}
\hline Time points & N & \multicolumn{2}{|l|}{$\begin{array}{l}\text { TMD change } \\
\text { between time points }\end{array}$} & \multicolumn{2}{l|}{$\begin{array}{l}\text { TMD correlations between } \\
\text { time points }\end{array}$} \\
\hline & & M-diff & SD & $\begin{array}{l}\text { Correlation } \\
\text { statistic }(\mathbf{r})\end{array}$ & $\begin{array}{l}\text { Significance } \\
(\mathbf{p} \text {-value) }\end{array}$ \\
\hline Mobilisation $\rightarrow$ mid-mission & 168 & +2.2 & 6.7 & .518 & $<.001$ \\
\hline Mid-mission $\rightarrow$ demobilisation & 168 & +0.1 & 5.8 & .677 & $<.001$ \\
\hline Mobilisation $\rightarrow$ demobilisation & 168 & +2.2 & 6.5 & .430 & $<.001$ \\
\hline
\end{tabular}

Table 2. Stability of ER profiles across three time points. 
BRUMS profiles at mobilisation appear to predict ER both during and towards the end of missions. A previous study demonstrated that ER profiles developed during demobilisation predict adaptation six weeks after the mission $(\mathrm{N}=181, \mathrm{r}=.335, \mathrm{p}$ $=.002) \cdot{ }^{20}$ Furthermore, elevated BRUMS scores predicted self-report post-traumatic stress symptoms six weeks after an active interdiction operation $(\mathrm{r}=.399, \mathrm{p}<.01){ }^{20}$

Referring to Assumption 3, the role of dispositional versus situational factors not all maritime operations are equal, with different operational tempos and temporal duration across various deployments reported. This raises the question whether the framework will hold across different mission parameters. The effect of mission circumstances was investigated by entering three different mission parameters - namely type of platform (e.g. large ships vs small ships), type of mission (e.g. monitoring patrol vs active maritime interdiction), and mission duration (e.g. 6 weeks vs 4 months) - into an analysis of sailors' ER profiles. When comparing scores across six separate missions over the four years (2015-2019) (using ANOVA), no significant differences in ER profiles were found between different deployments $\left(\mathrm{F}_{4,594}=.890, \mathrm{p}=.470\right)$. This finding is supported by studies in other ICE environments, ${ }^{125,126}$ and suggests that operational adaptation in ICE environments may rely on intra-personal factors, such as ER, rather than on external circumstances, such as mission duration.

The effects of one dispositional factor, namely sailor resiliency, are presented in Table 3. The BSRS predicted ER profiles at all three time points, which is also supported by data from other sources, ${ }^{3}$ further suggesting that operational adaptation in ICE environments is also reliant on dispositional factors.

\begin{tabular}{|l|l|l|l|}
\hline Time frame & $\mathbf{N}$ & Correlation $(\mathbf{r})$ & $\begin{array}{l}\text { Significance } \\
(\mathbf{p}-\mathbf{v a l u e})\end{array}$ \\
\hline BSRS $\rightarrow$ mobilisation & 390 & -.459 & $<.001$ \\
\hline BSRS $\rightarrow$ mid-mission $( \pm 6$ weeks $)$ & 222 & -.509 & $<.001$ \\
\hline BSRS $\rightarrow$ demobilisation $( \pm 14$ weeks $)$ & 200 & -.392 & $<.001$ \\
\hline
\end{tabular}

Table 3. Correlation between BSRS total score and ER profiles at three time points.

Normative ER reference data relating to Assumption 4 - availability of populationspecific norms - can be found in Table 1. Population-specific reference data are now available to support ethical interpretation for future use of BRUMS scores for practical purposes.

\section{Discussion, limitations and recommendations}

This article presented the move by the IMM to re-orientate mobilisation and demobilisation programmes towards a P\&P approach to PA during and after operational missions. Productive utilisation of this framework - that adaptive ER can reliably account for effective PA, and can be predicted in ICE contexts - is contingent on a number of assumptions being met. 
Four assumptions were identified and considered. Empirical support was found for Assumption 1, in the absence of debilitating clinical psychopathology in the sample tested. Support was also found for Assumption 2, in the temporal stability as well as predictability of the ER measure across different time points. Assumption 3 was supported by data that indicated that the dispositional factor was a small but statistically significant predictor, while the situational factors that were tested had little predictive value on ER. Lastly, population-specific reference data for the SAN Fleet are now available in support of Assumption 4.

The outcome of health support in the military environment is often measured against the rate of medical casualties. Within an approach where PA is promoted, outcome could potentially be measured against the rate of psychological casualties (defined as a person who cannot fulfil his or her mission role because of primarily psychological-related difficulties). On ships, however, this becomes difficult to determine, as it is not always clear what would constitute a 'casualty' on board a ship at sea. For this article, casualty rates thus had to be calculated using estimates. ${ }^{127}$

In-mission psychological casualty rates for ship-based maritime operations have been estimated by dividing known psychological casualties by the number of sailors on a ship, per mission, over the period 2015-2019). This resulted in an estimated $0.2 \%$ in-mission psychological casualty rate.

Post-mission psychological casualty rates for ship-based maritime operations have been estimated by dividing the known cases of mental (ill)health subsequent (and at least superficially related) to specific missions, by the number of sailors on that mission, per mission, over the period 2015-2020). This resulted in an estimated $0.4 \%$ attrition rate due to pathological post-mission stress reactions.

Against the background of initial empirical support for the underlying assumptions to the approach of the IMM, their P\&P approach can with some confidence be considered for continuing implementation. The usefulness of the P\&P approach will have to be monitored against, inter alia, psychological casualty rates during missions, and mental health reports after missions.

\section{Limitations and future directions}

A major limitation to the data presented here in support of the proposed P\&P approach is the lack of objective and standardised PA indicators, such as reports from supervisors or peers, to verify and triangulate the efficacy of assessing ER as a marker of more, or less, effective PA. Future studies will need to include objective PA markers, which do not solely rely on self-report measures, e.g. third-party reports or even neurophysiological indicators, in order to investigate further the link between ER and PA, both on ships and after deployments. ${ }^{128}$

This study used a measure of dispositional resilience to evaluate trait effects on ER in this context, and it is acknowledged that trait resilience rarely explains more than a 
small portion of actual variance of ER across situations. Future studies may benefit from including other dispositional factors as well.

In addition, it is worth noting that the study on which this article is based, focused on a delineated conceptualisation and measurement of a component of PA, namely ER, in the maritime operational environment of the SAN. Ultimately, individual differences in PA remain a complex configuration of social, emotional, occupational and physical dimensions of adjustment to the peculiar stressors and circumstances of particular ICE contexts and samples. Some of these were identified recently in the Isolated and Confined Environments Questionnaire, which may hold promise for future research in the local SAN context. ${ }^{129}$

In conclusion, an approach focusing on the prediction and promotion of psychological adaptation, using the mobilisation and demobilisation of ship-based maritime operations, may be a useful mechanism to support enhanced personal performance and mental health resilience during and after missions. 


\section{ENDNOTES}

${ }^{97}$ Charles van Wijk is employed as a clinical psychologist at the Institute for Maritime Medicine. $\mathrm{He}$ is a keen diver, and his past research centred around both the neuropsychology of, and psychological adaptation in, hyperbaric environments, as well as supporting mental wellbeing in associated high-demand occupational settings. Current research interests are related to occupational mental health surveillance.

${ }^{98}$ Jarred H Martin, PhD, is a lecturer in the Department of Psychology at the University of Pretoria where he currently chairs the Master of Arts in Clinical Psychology degree programme. He served in the SANDF from 2012-2019, during which he was deployed with the South African Battalion of the United Nations' Force Intervention Brigade in the DRC, from 2014-2015. He also participated in various naval exercises and operations while stationed in Simon's Town. His research principally focuses on critical studies of gender and masculinity.

${ }^{99}$ DE Rohall, MW Segal \& DR Segal. "Examining the importance of organizational supports on family adjustment to army life in a period of increasing separation". Journal of Political and Military Sociology 27/1. 1999. 49-65.

${ }^{100}$ South African Military Health Service. South African Military Health Service: Conventional doctrine. Pretoria, 2008.

${ }^{101} \mathrm{CH}$ van Wijk \& JH Martin. "A brief sailor resiliency scale for the South African Navy". African Journal of Psychological Assessment 1. 2019. a12. doi: 10.4102/ajopa.v1i0.12

${ }^{102}$ P Suedfeld \& GD Steel. "The environmental psychology of capsule habitats". Annual Review of Psychology 51. 2000. 227-253. doi: 10.1146/annurev.psych.51.1.227

${ }^{103}$ EKE Gunderson. "Individual behavior in confined or isolated groups". In JE Rasmussen (ed), Man in isolation and confinement. Chicago, IL: Aldine, 1973, 145-164.

${ }^{104}$ LA Palinkas, EKE Gunderson, AW Holland, C Miller \& JC Johnson. "Predictors of behavior and performance in extreme environments: The Antarctic Space Analogue Program". Aviation, Space and Environmental Medicine 7/1. 2000. 619-625.

${ }^{105}$ AM Lane, C Beedie \& TJ Devonport. "Measurement issues in emotion and emotion regulation". In J Thatcher, M Jones \& D Lavallee (eds), Coping and emotion in sport. $2^{\text {nd }}$ ed. London: Taylor \& Francis, 2011, 79-101.

${ }^{106}$ N Eisenberg, RA Fabes, IK Guthrie \& M Reiser. "Dispositional emotionality and regulation: Their role in predicting quality of social functioning". Journal of Personality and Social Psychology 78. 2000. 136-157.

${ }^{107}$ J Gross (ed). Handbook of emotion regulation. New York, NY: Guilford Press, 2007.

${ }^{108}$ J Gross \& RA Thompson. "Emotion regulation: Conceptual foundations". In Gross ibid., pp. $3-26$.

${ }^{109}$ E van der Meulen, PG van der Velden, RCM van Aert \& MJPM van Veldhoven. "Longitudinal associations of psychological resilience with mental health and functioning among military personnel: A meta-analysis of prospective studies". Social Science \& Medicine 255. 2020. Art. 112814. doi: 10.1016/j.socscimed.2020.112814

${ }^{110} \mathrm{~S}$ Sanden, BH Johnsen, J Eid, et al. "Mental readiness for maritime international operation: Procedures developed by Norwegian navy". International Maritime Medicine 65. 2014. 1-5. doi: 10.5603/IMH.2014.0020

${ }^{111}$ DM McNair, JWP Heuchert \& E Shilony. Profile of mood states manual: Bibliography 1964-2002. New York, NY: Multi-Health Systems, 2003.

${ }^{112}$ PC Terry, AM Lane \& GJ Fogarty. "Construct validity of the POMS-A for use with adults". Psychology of Sport and Exercise 4. 2003. 125-139. 
${ }^{113}$ PC Terry, AM Lane, HJ Lane \& L Keohane. "Development and validation of a mood measure for adolescents". Journal of Sports Sciences 17. 1999. 861-872.

${ }^{114}$ AM Lane, A Jackson \& PC Terry. "Preferred modality influences on exercise induced mood changes". Journal of Sports Science and Medicine 4. 2005. 195-200.

${ }^{115}$ CR Pedlar, AM Lane, JC Lloyd, J Dawson, S Emegbo \& GP Whyte. "Sleep profiles and mood states during an expedition to the South Pole". Wilderness and Environmental Medicine 18. 2006. 127-132.

${ }^{116}$ RC Thelwell, AM Lane \& NJV Weston. "Mood states, self-set goals, self-efficacy and performance in academic examinations". Personality and Individual Differences 42/3. 2007. $573-583$.

${ }^{117} \mathrm{CH}$ van Wijk. "The Brunel Mood Scale: A South African norm study". South African Journal of Psychiatry 17/2. 2011. 44-54.

${ }^{118} \mathrm{CH}$ van Wijk, JH Martin \& C Hans-Arendse. "Clinical utility of the BRUMS in screening for post-traumatic stress risk in a military population”. Military Medicine 178/4. 2013. 372-376.

${ }^{119} \mathrm{CH}$ van Wijk, JH Martin \& WAJ Meintjes. "Diving under the influence: Issues in researching personality and inert gas narcosis". International Maritime Health 68/1. 2017. 52-59. doi: 10.5603/IMH.2017.009

${ }^{120}$ PC Terry, JR Potgieter \& GJ Fogarty. "The Stellenbosch Mood Scale: A dual-language measure of mood”. International Journal of Sport and Exercise Psychology 1/3. 2003. 231-245.

${ }^{121}$ GE Richardson. "The meta-theory of resilience and resiliency". Journal of Clinical Psychology 58/3. 2002. 307-321.

${ }^{122}$ E Sagone \& ME de Caroli. "A correlational study on dispositional resilience, psychological well-being, and coping strategies in university students". American Journal of Educational Research 2/7. 2014. 463-471.

${ }^{123}$ SS Luthar \& PJ Brown. "Maximizing resilience through diverse levels of inquiry: Prevailing paradigms, possibilities, and priorities for the future". Developmental Psychopathology 19/3. 2007. 931-955. doi: 10.1017/S0954579407000454

${ }^{124}$ SR Maddi. "The history of hardiness: Twenty years of theorizing, research, and practice". Consulting Psychology Journal 54/3. 2002. 173-85. doi: 10.1037/1061-4087.54.3.173

${ }^{125}$ LA Palinkas. "The psychology of isolated and confined environments: Understanding human behavior in Antarctica". American Psychologist 58/5. 2003. 353-363.

${ }^{126}$ GM Sandal. "Coping in Antarctica: Is it possible to generalize results across settings?" Aviation, Space and Environmental Medicine 71. Suppl. 9. 2000. A37-A43.

${ }^{127}$ Most of the figures used for these calculations cannot be substantiated (they were inferred from various post-mission reports, medical data, local experience at IMM, or are classified and therefore not accessible), and are therefore estimations only.

${ }^{128}$ C Sargent, C Gebruers \& J O’Mahony. “A review of the physiological and psychological health and wellbeing of naval service personnel and the modalities used for monitoring”. Military Medical Research 4. 2017. Art. 1. doi: 10.1186/s40779-016-0112-3

${ }^{129}$ M Nicolas, G Martinent, P Suedfeld \& M Gaudino. "Assessing psychological adaptation during polar winter-overs: The isolated and confined environments questionnaire (ICE-Q)". Journal of Environmental Psychology 65. 2019. Art. 101317. doi: 10.1016/j.jenvp.2019.101317 